\title{
Tongue Characteristics of Different TCM Syndromes on Asthma
}

\author{
Sijing WANG \\ Basic Medical College \\ Shanghai University of Traditional Chinese Medicine \\ Shanghai, China \\ e-mail: 1158278555@qq.com
}

\author{
Zhaoxia XU* \\ Basic Medical College \\ Shanghai University of Traditional Chinese Medicine \\ Shanghai, China \\ e-mail: Zhaoxia7001@126.com
}

\author{
Yiqin WANG \\ Basic Medical College \\ Shanghai University of Traditional Chinese Medicine \\ Shanghai, China \\ e-mail: Wangyiqin2380@vip.sina.com
}

\begin{abstract}
Asthma is kind of disease affecting quality of life. Different TCM asthma types have different characteristics. TCM tongue images can be objective indexes for asthma TCM differentiation and therapeutic evaluation. More and more modern technique and analysis methods are used to discuss meaning of tongue. In this review, we hope it can be helpful to comb the process of TCM tongue research.
\end{abstract}

\section{Keywords-asthma; TCM syndromes; tongue characteristics}

\section{INTRODUCTION}

Asthma is a serious global health problem affecting all age groups. Its prevalence is increasing in many countries, especially among children. A 'new' definition of asthma identifies its heterogeneous nature, chronic airway inflammation, and the core clinical features of variable symptoms and variable expiratory airflow limitation [1]. The occurance and development of asthma have close correlation with host factors and environmental factors. Coating and nature of tongue can reflect variances of pathogenic factor and righteousness. And this information can be used to give references to prevention and treatment of bronchial asthma. Therefore, tongue observation is a preferable objective index for asthma [2].

\section{LITERATURE RESEARCH OF TONGUE}

G Chujie [3] did research on literature published on the VIP database from 1989 to 2011.He found 3131 cough variant asthma (CVA)-related literatures and 67 of them were in accordance with criteria. The result is that main tongue characteristics was red tongue (33.32\%), reddish tongue $(28.64 \%)$, pale tongue $(20.76 \%)$.Tongue with thin (27.99\%), white (25.97\%) tongue coating were most common, followed by yellow fur (16.91\%),greasy tongue fur $(13.85 \%)$. This research suggests that excess pathogenic factors is important for CVA.

L Xiaosheng et al. [4] collected ancient 350 case records. Based on smallest knowledge unit to annotate and standardize four diagnostic methods information, they construct the asthma cases data warehouse. They found that white coating is the highest frequency, accounting for $39.14 \%$, followed by thin fur, greasy fur, yellow fur and thick fur. Red tongue is highest frequency, accounting for $20.29 \%$, followed by pale tongue, dark red tongue, enlarged tongue. The rule of TCM diagnosing could be uncovered with data mining technique.

Z li [5] applied date mining on 175 child CVA medical records diagnosed and treated by Professor An Xiaoxian. She found the tongue feature is that among all visits red tongue accounting for $82 \%$, followed by reddish tongue accounting for $10 \%$. The most common tongue coating is white. Main TCM syndromes are Wind Evil Encroach Lung-type and Lung Heat-type. Through data mining technique can make veteran TCM doctors' clinical experience visualized.

$\mathrm{Z}$ Licheng et al [6] investigated the role of association rules in analysising TCM medical records. He found some correspondence between tongue information and TCM syndromes. The probability that asthmatic patients with throat phlegm sound and thin coating located in lung is 87.5\%.The rate that patients with TCM phlegm evil who have phlegm and thin coating is $88.89 \%$.The rate of adverse rising of pulmonary qi-type of patients who have throat phlegm sound and thin coating is $87.5 \%$.This research suggests that tongue information have a role in the presentation of location of disease and TCM syndromes.

\section{CHILDREN TONGUE FEATURES OF ASTHMA}

W Ming [7] uses DBASEIII software to build database of 700 asthmatic children medical records. The result is that according to the classification of cold and heat children can be divided into cold-type asthma (147/700) and heat-type asthma (553/700). And according to the classification of deficiency and excess children can be divided into Lung qi deficiency-type (416/700), Lung yin deficiency-type (86/700), Deficiency of lung and spleen-type (183/700), Deficiency of lung, spleen and kidney (15/700). Geographic tongue and incomplete loss of the tongue coating is accounting for $15.9 \%$.Among them, asthma because of 
external cause is accounting for $6.6 \%$,asthma because of internal cause is accounting for $2.5 \%$,mixed-type asthma is accounting for $5.8 \%$. The rate of childhood extrinsic asthma is higher than intrinsic asthma. It suggests that tongue conditions might be relative to allergic constitution.

$\mathrm{Z}$ Xia et al. [8] discussed children constitution of asthma. Zhao found that the tongue of Spleen and Kidney Deficiency Type I or Lung and Kidney Deficiency Type I is red with little coating, no coating or exfoliated tongue coating. And the tongue of Spleen and Kidney Deficiency Type II or Lung and Kidney Deficiency Type II is pale tongue, white coating, greasy coating, thick and greasy coating. This suggests that tongue conditions might be relative to children deficiency constitution.

Y Rong [9] observed 132 children tongue of asthma and found main feature of lingual diagnosis is pale tongue, thin and white tongue coating (35/132), pale tongue, white and greasy coating (28/132). All TCM syndromes are excess -type. They can be divided into cold-type asthma (67/132), Heat-type asthma (28/132), and Phlegm-type asthma (43/132). It suggests that tongue is relative to phlegm-evil and cold-evil.

N Yanliu [10] observed TCM symptoms distribution of 120 CVA children. He found that the rate of red tongue is high, accounting for $63.33 \%$.Main TCM syndromes of CVA children are Yin deficiency and phlegm dampness-type and Qi-Yin deficiency. Tongue of Yin deficiency and phlegm dampness-type is red tongue, thin and white coating. Tongue of Qi-Yin deficiency is red tongue or reddish tongue, thin and white coating. It suggests that red tongue is external representation of children's Yin deficiency constitution.

$\mathrm{T}$ Jingyi [11] made frequency statistic and cluster analysis on 214 cases of asthmatic children who were in remission stage. It turned out that thin and white coating (67.8\%), pale-enlarged tongue $(45.3 \%)$, pale tongue (32.2\%), red tongue with little fluid (30.8\%) are the main tongue. Through using $\mathrm{k}$-means clustering and system clustering method on analysing symptoms, she found that thin and white coating and other symptoms were concluded in Cluster 1.It suggested that it appears more in asthma remission stage. After applying principal component analysis on symptoms, it turned out that component coefficient of thin and white coating and pale-enlarged tongue and other symptoms is higher than 0.5.It demonstrated that this kind of symptoms are scattered extensively in asthma remission stage of children. And correlation analysis of symptoms and TCM syndromes demonstrated a correlation between thin and white coating, pale-enlarged tongue and lung-spleen qi deficiency syndrome. There was a high correlation between exfoliated tongue coating, red tongue with little fluid and Yin-deficiency of lung and kidney syndrome. Pale tongue is correlated to splenonephric Yang-deficiency.

\section{THERAPEUTIC EVALUATION OF CHILDREN ASTHMA}

W Ningru et al. [12] observed clinical therapeutic effect of Small Qinglong Decoction in the treatment of acute exacerbation bronchial asthma of cold syndrome. Nature and coating of tongue were concluded into evaluating indicators. Normal tongue was recorded as 0 point. Pale tongue was recorded as 1 point. And 0 point means normal coating, 1 point means white coating, 2 point means white greasy coating. There was no significant difference on nature of tongue between observational group and control group after treatment $(\mathrm{P}>0.05)$. However, there is significant difference on tongue coating between observational group and control group after treatment $(\mathrm{P}<0.05)$.Xiao Qinglong decoction could improve tongue coating of asthmatic children. No significant difference found in nature of tongue between observational group and control group might due to that course of treatment is just three weeks [12].

$\mathrm{Z}$ Chunqiu et al.[13]investigated clinical therapeutic effect of JianPiYiqiXiaoJi Decoction in treatment of children combined with asthmatic children combined with respiratory infection(RRI).The TCM syndrome of this kind of children can be classified as Lung and Spleen Qi Deficiency-type combined with accumulation of phlegm-heat. Tongues were recorded as 0 point, 1 point and 2 point from normal to abnormal. The result turned out that control group was superior to observational group in improving tongue. This research suggested that JianPiYiQiXiaoJi decoction can improve symptoms including tongue of asthmatic children.

L Bing et al. [14] collected medical records of 150 normal children and 150 asthmatic children. Then he divided asthmatic children into TCM treatment group (A group) and western medicine treatment group (B group). Normal children were chosen as observational group(C group).Tongue images of three groups were recorded by TCM tongue instrument. Then these quantized images were analysised to find regular pattern of phlegm dampness constitution and damp-heat constitution. IgE were also tested. It turned out that the composition ratio of A group yellow coating, thick coating and greasy coating was decreasing along with time going by. The ratio of phlegm dampness constitution and damp-heat constitution of A, B group were also decreasing. There was no significant decline found in $\mathrm{C}$ group. IgE of group $\mathrm{A}$ and $\mathrm{B}$ was much lower than themselves after treatment $(\mathrm{P}<0.01)$. There was no significant difference between Group A and Group B. It suggested that tongue could reflect change of asthmatic children constitution.

\section{V.Adult Tongue FeAtures of Asthma}

W Shiting et al. [15] select tongue parameters from 108 asthma patients. It turned out that main tongue were red tongue and reddish tongue. The most common tongue coating is white coating, yellow coating and thin coating .Among different TCM syndromes, the tongue color parameter, tongue coating parameter, thick tongue coating parameter, and enlarged or thin tongue body showed significant difference. These parameters may work as a reference for TCM syndrome differentiation.

Z Zhaowang [16] collected 1500 medicial records of asthmatic patients from 17 hospitals in 5 provinces. The result analyzed by logistic regression method demonstrated 
that white tongue coating was related to external cold and interior phlegm-retention-type $(\mathrm{B}=0.993, \quad \mathrm{OR}=4.701)$. Yellow and greasy tongue coating was related to phlegm-heat obstructing in the lung-type $(B=1.946$, $\mathrm{OR}=9.033)$. White and greasy tongue coating was related to turbid phlegm obstructing in the lung-type $(B=0.580$, $\mathrm{OR}=1.018$ ). Pale tongue was related to Lung qi deficiency $(B=0.909, O R=2.790)$. Spleen qi deficiency-type $(B=2.207$, $\mathrm{OR}=1.132)$ and Kidney qi deficiency $(\mathrm{B}=0.910, \mathrm{OR}=2.703)$.

$S$ Suofang [17] collected four diagnostic information of 430 asthmatic patients.

These information were analysised by CFA of Amos 4.0.Regression coefficient 0.4 was selected as boundary value of primary symptom and secondary sympton. Factor 4 was concluded as phlegm-heat obstructing in the lung-type. The tongue of it was yellow coating. Factor 6 was concluded as syndrome of intermingled deficiency and excess. The tongue was red tongue and greasy tongue coating. The correlation coefficient between Factor 4 and Factor 6 was 0.890.It suggests that syndrome of intermingled deficiency and excess-type was changed from phlegm-heat obstructing in the lung-type. The change of tongue also confirmed that [17].

S Huiyuan [18] used MDR version 2.0 software to analysis 365 medical records of asthmatic patients who were in chronicity-persistent period. It turned out that thick coating was closely related to speak less and lack of strength for who were in Deficiency of lung and spleen. Thick coating was also related to deep pulse and fatigue. Greasy tongue coating and lack of strength had obviously positive correlation for who were in turbid phlegm obstructing in the lung-type. It suggests that MDR has advantage in dealing with high-order data.

L Wen [19] collected 217 TCM records of asthmatic patients who were in acute exacerbation. Cold-type, Heat-type, Anemophlegmatic-type and Deficiency-type asthma analysed by cluster method were basic TCM syndrome type. Tongue of Cold-type asthma was white tongue coating, reddish tongue and cyanose sublingual vessel. Tongue of Heat-type asthma was yellow tongue coating, red tongue and swelling sublingual vessel. Tongue of anemophlegmatic-type ashma was thin and white tongue coating, reddish or enlarged tongue and cyanose sublingual vessel. Tongue of deficiency-type asthma was swelling sublingual vessel. Due to tongue petechiae appeared in Four type of asthma, it suggests that stasis of blood was along with asthma.

\section{CORRELATION BETWEEN TONGUE AND PHYSICOCHEMICAL INDEXES}

Z Shanshan et al. [20] collected tongue coating of children patients including AAPs (asthmatic patients). They found that WBC count of yellow tongue coating was higher than that of white tongue coating. The total number of peripheral blood WBC for yellow coating patients was also higher than that of white coating patients. It suggests that yellow tongue coating is related to inflammation infection.

$\mathrm{Z}$ bojun et al. [21] observed AAPs' microcirculation of nail fold and tongue tips from different TCM syndromes.
They found nail fold microcirulation weighted integral of Lung-qi deficiency and Lung Kidney-qi deficiency was relatively low in 132 patients. It illustrated that change of blood flowing state of these types were light. Flow state change of lung and kidney Qi asthenia and yin deficiency-type became aggravated. Flow state change of lung and kidney Yin asthenia and yang deficiency-type was the most acute. Micro thrombosis could be seen in this type. Microcirulation observation of tongue tip was done in 36 patients. They found that pale teeth-marked tongue showed clavate papillae was enlarged, pale blood color and unclear flow state. Red tongue and thin tongue body showed clavate papillae was shrunk, dark blood color and slow flow stage. Dark and purple tongue showed special-shaped clavate papillae, more blood-stasis vessel and slow flow stage. This research indicated association between tongue image and change of microcirulation.

L Xueliang et al. [22] observed change of four diagnostic information, TCM syndromes and lung function of asthmatic patients who were in remission stage. R,G, B values of tongue tip, left-side, right-side, whole-body and G value of tongue root coating color showed significant rise in asthmatic patients after TCM treatment $(\mathrm{P}<0.05)$. Other symptoms also had improvement. Objective test of TCM four diagnostic methods can be effective basis for TCM syndrome diagnosis, observation of disease evolution and therapeutic evaluation.

\section{SUMMARY AND PROSPECT}

There are a large amount of descriptions about tongue of asthma in ancient TCM records. Nowadays, researchers explore some regular pattern of asthmatic tongue and TCM syndromes using computer technique. However, tongue observation relies on subjective judgment and it is influenced by knowledge levels and clinical experiences of doctors. And this information shows less objectivity. Therefore, some researchers use tongue image instrument to record and analyze tongue and gained some results. We need to do larger sample and objective test on tongue. We can also record dynamic rules of tongue image.

\section{ACKNOWLEDGMENT}

This work is supported by the Shanghai Natural Science Foundation (No.14ZR441500) and the Shanghai TCM Special Funds Project of Health and Family Planning (No.2014JP029A).

\section{REFERENCES}

[1] Global Initiative for Asthma (GINA), Global Strategy for Asthma Management and Prevention. Available From www.ginasthma.org.

[2] W Petian, "What is the meaning of tongue observation in asthma treatment?", Journal of Traditioal Chinese Medicine, vol.45, Oct.2004, pp.793-794.dol:10.3321/j.issn:1001-1668.2004.10.042.

[3] G Chujie, "A research for Syndrome Characteristics of Cough Variant Asthma," Guangzhou University of Traditional Chinese Medicine, 2012.

[4] L Xiaosheng,Z Licheng, W Xinkai,L Seqi,Z Qinglin, “ Based on Data Warehouse Technique to Uncover the Rule of Treating and Diagnosing Asthma from Incunabula TCM Medical Records, 
"Journal of Jiangxi University of Traditional Chinese Medicine, vol.24,Jun.2012,pp.13-22.dol:10.3969/j.issn.1005-9431.2012.03.004.

[5] Z Li, "A research on Clinical Experiences from CVA Children Diagnosed and Treated by Professor A Xiaoxian based on Data Mining Technique," China Academy of Traditional Chinese medicine, 2014.

[6] Z Licheng,X Hanrong,L Seqi,Z Qinglin, Z Qiming, Lu Aiping, "The Association Analysis in 445 Case of Famous Practitioner of Chinese Medicine about Asthma, "Journal of Jiangxi University of Traditional Chinese Medicine,vol.19,Oct.2007, pp.83-87, dol:10.3969/j.issn.1005-9431.2007.05.032.

[7] W Min, "Statistical data analysis of 700 asthmatic children on state of illness and TCM Syndrome Differentiation," Shanghai Journal of Traditional Chinese Medicine, No.6, Jun.1992, pp.25,dol:10.16305/j.1007-1334.1992.06.013

[8] Z Xia,S Shurong, "Constitution Investigation and Typing on 100 Cases of Asthma Children, "Journal of Chengdu University of Traditional Chinese Medicine, vol.24, Jun.2001, pp.16-17. dol:10.3969/j.issn.1004-0668.2001.03.007.

[9] Y Rong, “Analysis on TCM Syndrome Differentiation of 132 asthmatic children, "Journal of Pediatrics of Traditional Chinese Medicine, vol.7, Jan. 2011, pp.30-31. dol:10.3969/j.issn.1673-4297.2011.01.011.

[10] N Yanliu, "In 120 cases of children with cough variant asthma Analysis of TCM syndrome, "China Academy of Traditional Chinese medicine, 2012

[11] T Jingyi, "Children Bronchial Asthma In Remission Stage of The Distribution Regularity of TCM Syndromes And JIANPIBUFEIHUATANFANG with Experimental Study of The Expression of Rat PDGF,TGF- $\beta 1$ and c-cosmRNA," Anhui University of Traditional Chinese Medicine, 2015.

[12] W Ningru, H He,F Yinglan, Z Yafeng, "Curative Effect Observation of Small Qinglong Decoction in Treatment of Cold Type of Bronchial Asthma, "Chinese Archives of Traditional Chinese Medicine, vol.33, Dec. 2015, pp.2912-2915. dol:10.13193/j.issn.1673-7717.2015.12.026

[13] Z Chunqiu,"Randomized Controlled Clinical Study of Jian Pi Yi Qi Xiao Ji Tang in Bronchial Asthmatic Children Combined with Recurrent Respiratory Infection, "Journal of Practical Traditional Chinese Internal Medicine, vol.26, Apr.2012, pp.29-30, dol: 10. 3969 /j. issn. 1671 - 7813. 2012. 04. 15

[14] L Bing, "Correlation among Tongue Picture, Constitution and level of Serum IgE in Children with asthma, "Jinlin Journal of Traditional Chinese Medicine, vol.33, Aug.2013, pp.809-811. dol:10.3969/j.issn.1003-5699.2013.08.026.

[15] W Shiting, Z Xiaodan, H Yiming,W Yiqing, “TCM Characteristic Analysis of 108 Patients' Tongue and Pulse Parameters by the TP-1 Digital Analysis Instrument, "World Science and Technology -Modernization of Traditional Chinese Medicine and Materia Medica,vol.11,Jun.2009,pp:806-809.dol:10.3969/j.issn.1674-3849.20 09.06.009.

[16] Z Zhaowang, "Clinical investigation of the distribution of syndromes acute exacerbation of bronchial asthma," Henan College of Traditional Chinese Medicine, 2014.

[17] S Suofang,L Xiufang.Y Zhilin,W Daocheng,K Xiangwen,S Zhencang,Chen Qiguang,M jie, "Investigation and confirmatory factor analysis of information collected with the four diagnostic methods in patients with bronchial asthma." Journal of Integrative Medicine, vol.3, Sep.2005, pp.363-365.

[18] S Huiyuan, "TCM syndrome features of Chronic Persistent Asthma and Application Research based on 'Lung-Spleen viscera syndrome differentiation '.” [D]Beijing University of Traditional Medicine, 2015

[19] L Wen, "Research on TCM Syndrome Distribution and Pathogenesis of adult bronchial asthma, "Guangzhou University of Chinese Medicine, 2011.

[20] Z Shanshan,Z Xiqin, "Observation of tongue coating and peripheral blood WBC of children with common disease," Journal of Nanjing University of Traditional Chinese Medicine, No.1,1986,dol:10.14148/j.issn.1672-0482.1986.01.024

[21] Z Bojun,C Chao,X Wenbin, "Association between Peripheral microcirculation and Different TCM asthma Syndromes,” Journal of Chinese Microcirculation,No.3,1996,pp.159-161.

[22] L Xueliang, X Zhaoxia, W Yiqin, G Rui, H Yiming,L Zhao,X Jin. "Clinical Evaluation on Patients with Bronchial Asthma in Remission Based on Four Diagnostic Information,” World Science and Technology-Modernization of Traditional Chinese Medicine and Materia Medica, vol.16, Jun.2014, pp.1294-1299. dol:10.11842/wst.2014.06.016. 